\title{
Spectral sensitivity of the visual system of endemic Baikal amphipods
}

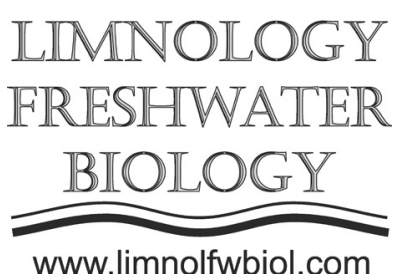

\author{
Drozdova P.B. ${ }^{1,2 *}$, Saranchina A.E. ${ }^{1 * *}$, Timofeyev M.A. ${ }^{1,2}$ \\ ${ }^{1}$ Institute of Biology, Irkutsk State University, Lenin str. 3, Irkutsk, 664025, Russia \\ ${ }^{2}$ Baikal Research Centre, Lenin str. 21, Irkutsk, 664003, Russia
}

\begin{abstract}
In this work, we attempted to explore the visual system of Baikal endemic amphipods with behavioral experiments. These data show that a littoral species Eulimnogammarus cyaneus can sense most wavelengths in the human-visible range, while in the case of a deep-water species Ommatogammarus flavus we did not see any response to light.
\end{abstract}

Keywords: Baikal, Amphipoda, vision, opsins, evolution

Lake Baikal, the oldest, largest and deepest lake on the planet, hosts an amazing variety of endemic species. Among them, there are several prominent species flocks including sculpins and amphipods (Cristescu et al., 2010). Baikal sculpins (Pisces: Cottidae) include 33 species (Goto et al., 2015), while Baikal amphipods (Crustacea: Malacostraca: Amphipoda: Gammaridae) include over 350 species and subspecies (Takhteev, 2019), and both taxa have representatives inhabiting all depths of the lake. The visual system of sculpins is wellstudied, and it is known that the maximum sensitivity of short wavelength-sensitive visual pigments shifts towards shorter wavelengths with increasing habitat depth (Bowmaker et al., 1994; Cowing et al., 2002). However, the data on the spectral sensitivity of Baikal amphipods, as well as other gammarids, is very scarce.

Thus, we attempted to infer the spectral sensitivity of amphipods from their behavior. Adult individuals of littoral Eulimnogammarus verrucosus (Gerstf., 1858) and E. cyaneus (Dyb., 1874), as well as deep-water Ommatogammarus flavus (Dyb., 1874), were used. The Eulimnogammarus species were collected by kick-sampling, and $O$. flavus individuals were collected with baited traps. The experiments were designed to test the known reaction of avoidance of light by amphipods (Stom et al., 2017) in two ways and held in a dark room. In the first group of experiments, we used a $60 \times 10 \times 10 \mathrm{~cm}$ glass tank covered with plastic food wrap to avoid small animals from accumulating in the corners and a fragment of a LED strip (emission peaks $457 / 520 / 593 / 626 \mathrm{~nm}$ ) or an infrared light source (emission peaks 835/953 nm) at one of the sides. After 1-5 minutes of subjecting the animals to the LED light source they were photographed, and the distribution of animal coordinates within the tank was used to judge if there was a behavioral response to light. In the second group of experiments, we placed one animal into a Petri plate with four sections labeled and illuminated its head. Then, we counted the number of times the animal changed the section within 2-5 minutes.

We found that $E$. cyaneus reproducibly avoided the light produced by all tested LED strips, with the blue strip having the most pronounced effect (Fig. 1A), but showed no reaction to infrared light. Moreover, if subjected to two light sources the animals avoided blue and green lights more intensively than the yellow and red ones (Fig. 1B). However, the blue LED was approximately two-fold more intensive than the red one. When the blue LED was covered with a filter allowing only $30 \%$ of the light passing through, the avoidance effect was less pronounced, and only two layers of the filter abolished the effect. Similarly, targeted illumination with blue or green LED triggered the most pronounced response in E. cyaneus and its congener E. verrucosus (Fig. 1C). Combined, these data suggest that littoral Baikal amphipods can sense most wavelengths in the human-visible range. However, in the case of $O$. flavus we did not find any response to any part of the visible light spectrum or infrared light (data not shown). The reasons behind this lack of response require further investigation.

Our analysis showed a prominent response to various wavelengths of visible light in a littoral amphipod species E. cyaneus and E. verrucosus. At the same time, a deep-water species $O$. flavus did not show any response to light. Further studies with other species would be interesting to characterize the diversity of visual systems in Baikal amphipods.

The work was supported by the Russian Science Foundation (grant \#19-74-00045).
* Corresponding author.
E-mail address: drozdovapb@gmail.com (P.B. Drozdova)
$*$ *Drozdova P.B. and Saranchina A.E. contributed equally to this work
(C) Author(s) 2020. This work is distributed under the Creative Commons Attribution 4.0 License. 

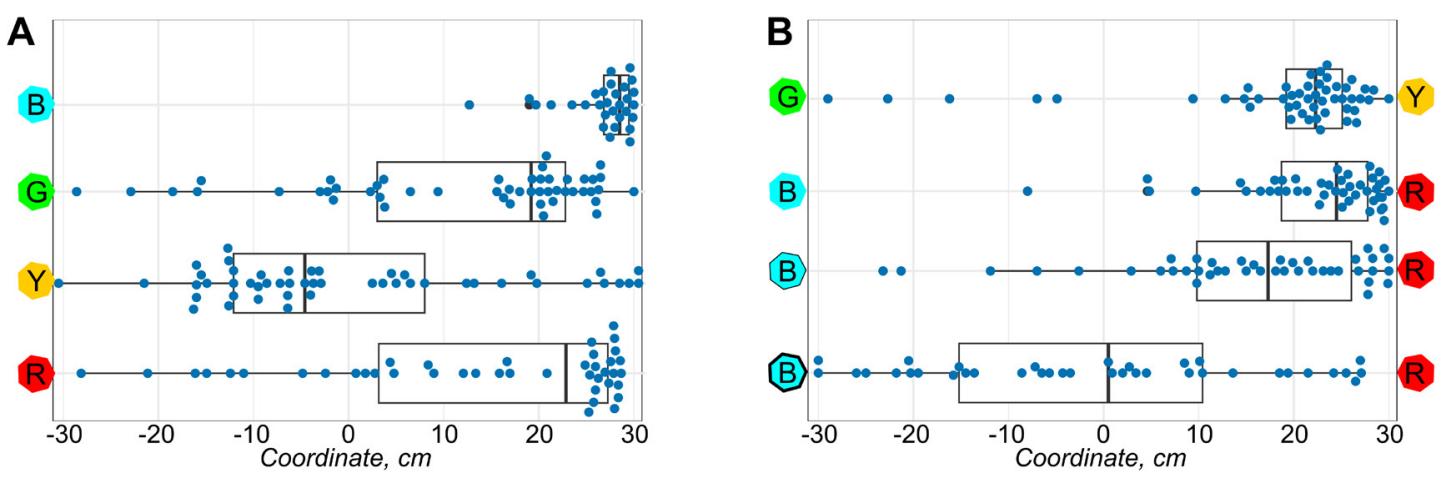

C
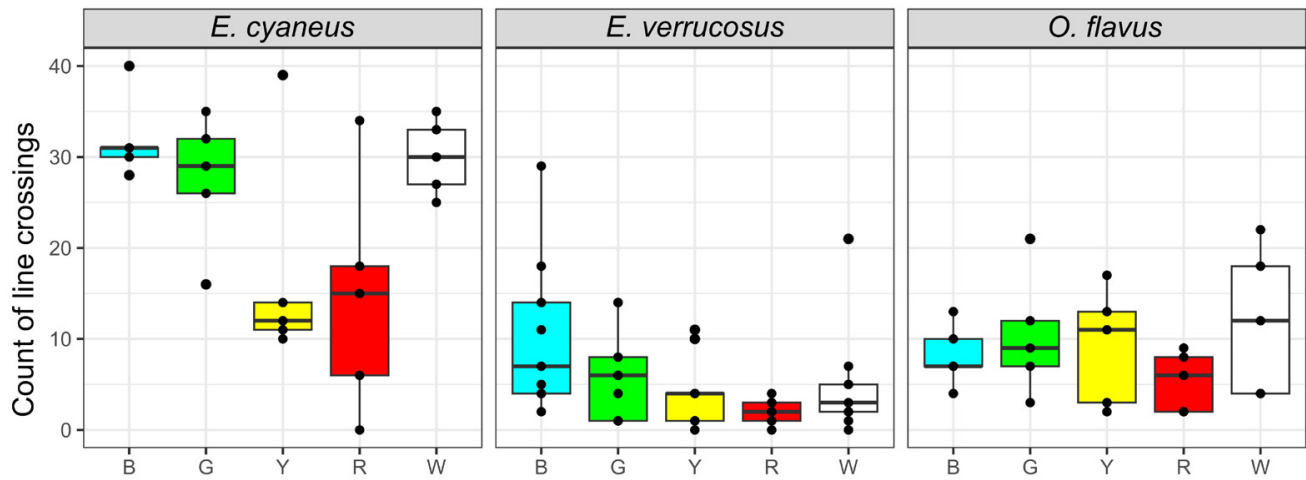

Fig.1. Behavioral response of Baikal amphipods to light. A, B: Avoidance of with LED lights by E. cyaneus. The thin and thick borders around the hexagon signify one and two layers of filter. C: Changes in locomotor activity of amphipods in response to targeted illumination of the head. B, blue LED; G, green LED; Y, yellow LED; R, red LED; W, white light.

\section{References}

Bowmaker J.K., Govardovskii V.I., Shukolyukov S.A. et al. 1994. Visual pigments and the photic environment: the cottoid fish of Lake Baikal. Vision Research 34: 591-605. DOI: 10.1016/0042-6989(94)90015-9

Cowing J.A., Poopalasundaram S., Wilkie S.E. et al. 2002. Spectral tuning and evolution of short wave-sensitive cone pigments in cottoid fish from Lake Baikal. Biochemistry 41: 6019-6025. DOI: 10.1021/bi025656e

Goto A., Yokoyama R., Sideleva V.G. 2015. Evolutionary diversification in freshwater sculpins (Cottoidea): a review of two major adaptive radiations. Environmental Biology of Fishes 98: 307-335. DOI: 10.1007/s10641-014-0262-7
Cristescu M.E., Adamowicz S.J., Vaillant J.J. et al. 2010. Ancient lakes revisited: from the ecology to the genetics of speciation. Molecular Ecology 19: 4837-4851. DOI: 10.1111/j.1365-294X.2010.04832.x

Stom D.I., Zhdanova G.O., Saksonov M.N. et al. 2017. Light avoidance in Baikalian amphipods as a test response to toxicants. Contemporary Problems of Ecology 10: 77-83. DOI: 10.1134/S1995425517010115

Takhteev VV. 2019. On the current state of taxonomy of the Baikal Lake amphipods (Crustacea: Amphipoda) and the typological ways of constructing their system. Arthropoda Selecta 28: 374-402. 